\title{
Service Ethnocentrism: A Conceptual Model (An Abstract)
}

\author{
Amro A. Maher and Tamer H. Elsharnouby
}

\begin{abstract}
The service marketing literature has recognized the importance of studying cross-cultural service encounters in which the service provider and the customer are from different cultures (Baker et al. 2008; Sharma et al. 2014; Maher and Sobh 2014). More recently, scholars have examined cross-cultural customercustomer interaction (CCI), which "is the active or passive interaction between two and more customers inside or outside the service setting. CCI may or may not involve verbal communication" (Johnson and Grier 2013, p. 306). The mere presence of one customer next to another is an example of such an interaction.

In this paper, we seek to contribute to this research stream on CCI by examining local residents' preference for consumption environments that are patronized by local residents versus foreign residents; we call this preference service ethnocentrism (SE). SE is similar to consumer ethnocentrism (Shimp and Sharma 1987) in that both involve an aversion to an object that is foreign, but the target of aversion is the service environment frequented by foreigners in the former and foreign products in the latter.

We conducted two focus groups with locals and a literature survey, which led us to develop our conceptual model. In this model, we propose to test the antecedents of SE. SE is defined as local residents' preference for consumption environments that are patronized by local residents versus foreign residents. In this model, we have identified factors that lead to an increase in SE and other factors that lead to a decrease in SE. Factors that lead to an increase in SE include feelings of comfort around locals, symbolic threat, realistic threat, and cultural distance from foreign residents. Factors that lead to a decrease in SE include feelings of comfort around foreign residents and cosmopolitanism.
\end{abstract}

References Available Upon Request

\author{
A.A. Maher $(\bowtie) \bullet$ T.H. Elsharnouby \\ Qatar University, Doha, Qatar \\ e-mail: amaher@qu.edu.qa; telsharnouby@qu.edu.qa
}

(C) Academy of Marketing Science 2017 